\title{
Erratum: Radiative Heat Transfer in Freestanding Silicon Nitride Membranes [Phys. Rev. Appl. 14, 024072 (2020)]
}

\author{
Chang Zhang, Alexandre Bouchard, Mathieu Giroux, Thea Abdul Nour, and Raphael St-Gelais* \\ Department of Mechanical Engineering, University of Ottawa, Ottawa, ON, Canada
}

(Received 5 July 2021; published 16 July 2021)

DOI: 10.1103/PhysRevApplied.16.019901

In our original work, we provided a closed-form heat transfer model for radiative and conductive heat transfer in SiN freestanding nanomechanical resonators. Here we identify and correct an error that slightly modifies calculated theoretical emissivity values for freestanding SiN films. In section III of our original manuscript, we used Kirchhoff's law $\left[\epsilon_{\lambda, \theta}(\lambda, \theta)=1-r_{\lambda, \theta}(\lambda, \theta)-t_{\lambda, \theta}(\lambda, \theta)\right]$ of thermal radiation with standard formalism for thin film interference for calculating the theoretical emissivity of a thin-film SiN membrane. The film amplitude reflection coefficient $r_{\lambda, \theta}(\lambda, \theta)$ was calculated as [1]:

$$
r_{\lambda, \theta}(\lambda, \theta)=r+\frac{t t^{\prime} r^{\prime} e^{i \delta}}{1-r^{\prime 2} e^{i \delta}}
$$

and the corresponding transmission coefficient $t_{\lambda, \theta}(\lambda, \theta)$ was:

$$
t_{\lambda, \theta}(\lambda, \theta)=\frac{t t^{\prime} e^{\frac{i \delta}{2}}}{1-r^{\prime 2} e^{i \delta}} .
$$

In this case, $r$ and $t$ denote reflection and transmission coefficients from fast to slow medium (i.e., from vacuum to $\mathrm{SiN}$ ) calculated from Fresnel's law, whereas $r^{\prime}$ and $t^{\prime}$ are the coefficients from slow to fast medium. The round-trip phase difference is:

$$
\delta=\frac{4 \pi n_{S i N} d \cos \theta_{t}}{\lambda},
$$

where $n_{S i N}$ is the complex refractive index of $\mathrm{SiN}, d$ is the film thickness and $\theta_{t}$ is the refraction angle in the film, expressed as a function of the incident angle $\left(\theta_{i}\right)$ using Snell's law.

In our original calculations, we mistakenly used the incident angle $\theta_{i}$ in lieu of $\theta_{t}$ in Eq. (3) when calculating $\delta$. This resulted in a $\sim 20 \%$ underestimation of the total hemispherical emissivity $\epsilon$ of SiN films. Original manuscript FIG 1, 3, and 4 are valid for all emissivity values and are therefore unaffected. Likewise, for our comparison between experiment and theory (i.e., original FIG 5 and associated discussion), this error is small relative to experimental uncertainties, such that the original conclusions stand.

FIG 2 and its associated discussion on the emissivity of SiN films is the only part of the manuscript that is noticeably affected; we therefore provide a corrected FIG 2 below (CORR FIG 2) with a brief updated discussion. In CORR FIG 2 (a), we now observe that the spectral normal emissivity $\epsilon_{\lambda, \theta}(\lambda, 0)$ is almost equal to the spectral hemispherical emissivity $\epsilon_{\lambda}(\lambda)$ that is obtained by integrating $\epsilon_{\lambda, \theta}$ over all angles using Lambert's cosine law. Therefore, in contrast with the original manuscript, we observe that a diffusive (i.e., angle-independent) assumption is an excellent approximation for the spectral hemispherical emissivity $\epsilon_{\lambda}(\lambda)$ of freestanding SiN membranes. We repeated the calculations used to produce CORR FIG 2 (a) for thicknesses ranging from $10 \mathrm{~nm}$ to $1 \mu \mathrm{m}$ and also noted excellent agreement. CORR FIG 2 (b - d) appear qualitatively similar to the original manuscript graphs, although their numerical values are now adjusted. In panel (d), we originally observed a plateauing effect, showing $\epsilon / d$ values that were essentially independent of film thickness for $\mathrm{d}<$ $100 \mathrm{~nm}$. This effect is not as pronounced anymore. Since the ratio $\epsilon / d$ dictates the fraction of heat transport occurring via radiation, we now observe that membranes are significantly more prone to radiation-dominated heat transport as thickness decreases, even for $d<100 \mathrm{~nm}$.

\footnotetext{
*raphael.stgelais@uottawa.ca
} 

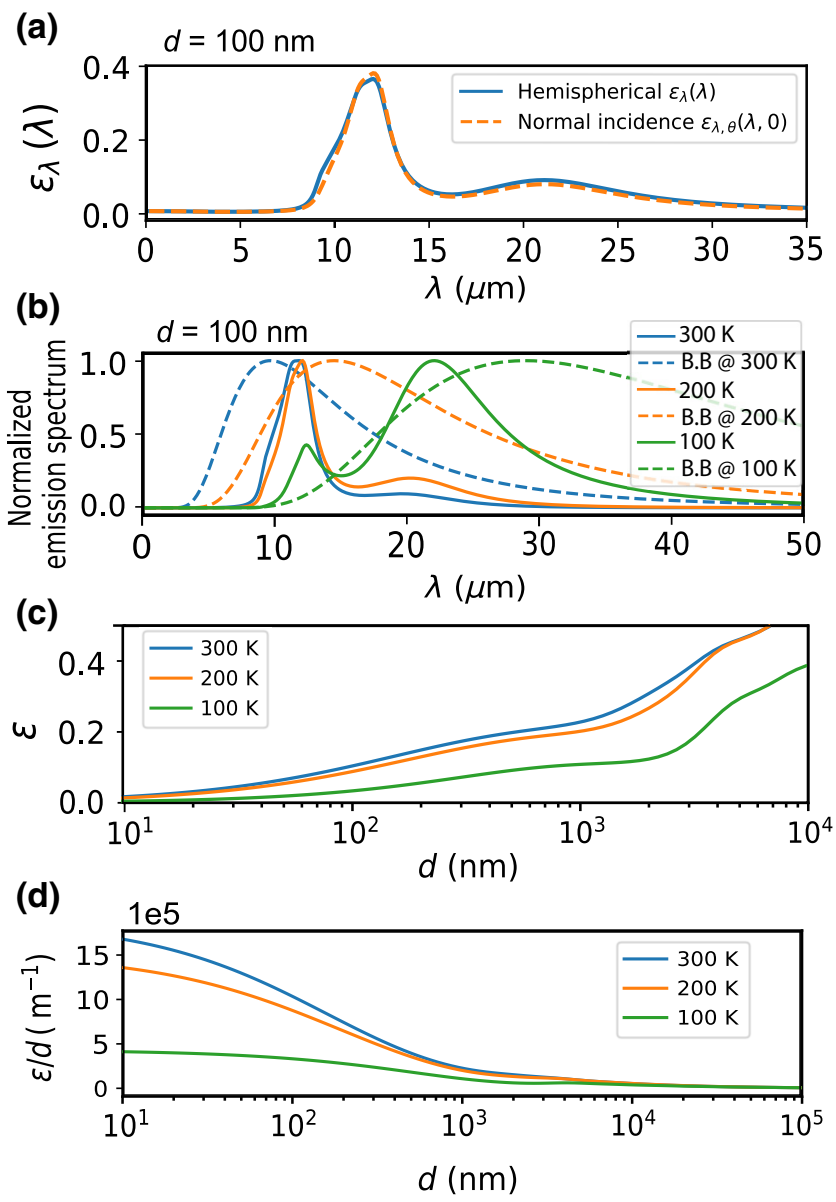

CORR FIG. 2. (a) Comparison between normal and hemispherical emissivity for freestanding SiN membranes. (b) Normalized emission spectrum of a 100-nm-thick SiN membrane at different temperatures. Dashed lines represent black-body (BB) spectra. As the temperature decreases, the peak of the emission spectrum moves toward longer wavelengths. (c) Hemispherical total emissivity of freestanding SiN membranes at different temperatures. (d) Hemispherical total emissivity normalized by film thicknesses for different temperatures. This quantity scales with the fraction of heat transfer occurring by radiation $\left(x_{r a d}\right)$ and is maximized for smaller membrane thicknesses. A typographic mistake in the y-axis label "1e5" was corrected from the original version.

[1] Frank L Pedrotti, Leno M Pedrotti, and Leno S Pedrotti, Introduction to Optics (Pearson, 2006), 3rd ed. 